\title{
Oral Administration of Docosahexaenoic Acid/Eicosapentaeinoic Acids Is Not Anticonvulsant in Rats: Implications for Translational Research
}

\author{
NICCOLO CURATOLO, CÉCILE LECOINTE, REGIS BORDET, LOUIS VALLÉE, CLAUDE GALABERT, PIERRE GRESSENS, \\ AND STÉPHANE AUVIN
}

Inserm U676, [N.C., C.L., P.G., S.A.], Université Paris 7, 75019 Paris, France; Service de Neurologie Pédiatrique [S.A.], Hôpital Robert Debré, 75019 Paris, France; Department of Pharmacology [R.B., L.V.], Institut de Médecine Prédictive et de Recherche Thérapeutique, Université de Lille-2, 59000 Lille, France; CERM [C.G.], Hôpital Renée Sabran, 83406 Giens Hyères, CHU Lyon, France

\begin{abstract}
Omega-3 and omega-6 poly-unsaturated fatty acids (PUFAs) are dietary fatty acids that are involved in a myriad of physiological processes in the brain. Although experimental data have shown that PUFAs have anticonvulsant properties, the outcomes of clinical trials have been controversial. Docosahexaenoic acid (DHA) is a PUFA which has been reported to exert anticonvulsant effects. Here we studied anticonvulsant potential of a mixture of enriched n-3 PUFA upon their oral administration in rats. We did not observe an anticonvulsant effect of n-3 PUFA in the i.v. pentylentetrazol threshold test. n-3 PUFA component was increased in the plasma of rats treated with the eicosapentaenoic acid (EPA)/DHA mix $(275 \mathrm{mg} / \mathrm{kg} / \mathrm{d} / 400 \mathrm{mg} / \mathrm{kg} / \mathrm{d})$ due to the increase of both DHA and EPA. We also found modification of PUFA composition in the brain. Despite PUFA profiles modified both in plasma and in the brain, we did not find any anticonvulsant effect of orally administered DHA. Further studies are needed to define the type and the amount of fatty acids that would possess anticonvulsant properties. As the existing literature suggests that the route of administration of PUFA may be crucial, future studies should involve oral administration to provide relevant clinical information. (Pediatr Res 70: 584-588, 2011)
\end{abstract}

$\mathrm{R}$ efractory epilepsy (i.e. persistence of seizures despite accurate diagnosis and carefully monitored treatment with antiepileptic drugs) has been reported in approximately $30 \%$ of epilepsy patients $(1,2)$. Therefore, new therapeutic approaches are required to effectively manage drug-resistant seizures. Ketogenic diet (KD) has been proven effective in epilepsy treatment in retrospective, prospective, and randomized controlled studies (3-6). While affording an opportunity for a dietary control of seizures in principle, $\mathrm{KD}$ is challenging as a long-term treatment regimen. The underlying mechanisms of the KD are currently unknown, but the role of caloric/ glucose restriction, ketone bodies, and polyunsaturated fatty acids (PUFAs) is under investigation.

Recent studies suggest that PUFA possess anticonvulsant properties, and they may be good candidates for nutritional

Received November 29, 2010; accepted July 6, 2011.

Correspondence: Stéphane Auvin, M.D., Ph.D., Service de Neurologie Pédiatrique et des Maladies Métaboliques, CHU Hôpital Robert Debré, 48, boulevard Sérurier, 75935 PARIS CEDEX 19-France; e-mail: auvin@invivo.edu

Supported in part by INSERM Grant (Contrat Interface INSERM 2010) [S.A.] and AP-HP Grant [P.G.].

The authors report no conflicts of interest. treatment of epilepsy (7). In experimental studies, PUFAs exert anticonvulsant effect in various seizure models, including pentylenetetrazol (PTZ) (8-11), kaïnate (12), iron chloride cortical application (9), electrical stimulation of the cortex (13), and audiogenic-seizures (9).

Several mechanisms have been proposed to explain the increase of seizure threshold by n-3 PUFA. One of the most common hypotheses involves modulating the function of voltage-dependent ion channels, particularly of the voltagedependent sodium channel (7) by a PUFA docosahexaenoic acid (C22:6n-3; DHA). This hypothesis was recently confirmed by the fact that s.c. injection of $400 \mathrm{mg} / \mathrm{kg}$ of DHA increased the latency of PTZ-induced seizures (14). Other putative mechanisms include binding of PUFA to peroxisome proliferator-activated receptor (PPAR)- $\alpha$ and/or PPAR $\gamma$, as well as anti-inflammatory effects of n-3 PUFAs (7).

Alpha-linolenic acid (ALA; C18:3n-3), eicosapentaenoic acid (EPA; C20:5n-3), and DHA have similar relative potencies in vitro $(13,15-17)$; however, of the three PUFA, only DHA is present in the brain in appreciable amounts. Although the uptake of linoleic acid (LA; C18:2n-6), ALA, and EPA by the brain is similar to that of DHA, the latter is preferentially incorporated into membrane phospholipids, whereas most of (i.e. $>90 \%$ ) of LA, ALA, and EPA are $\beta$-oxidized or recycled into other lipids (e.g. sterols and saturated fatty acids) $(18,19)$.

Despite the increased accumulation of experimental evidence, clinical studies of PUFA in epilepsy patients have been yielding conflicting results (20-22). Failure of PUFA to exert anticonvulsant effects in clinical trials may stem from several causes, such as the type of PUFA used, lack of data on the baseline PUFA intake, absence of reliable biomarker of PUFA, and dose-dependence effect of PUFA (7). Furthermore, all clinical trials involve EPA/DHA, while in the majority of preclinical studies anticonvulsant effects were estab-

\footnotetext{
Abbreviations: AA, arachidonic acid; ALA, alpha-linolenic acid; DHA, docosahexaenoic acid; D-0, day 0 (start of the diet); D-30, day 30 (last day on the diet, day of experimentation); EPA, eicosapentaenoic acid; KD, ketogenic diet; LA, linoleic acid; LCP, long chain polyunsaturated fatty acids; HPTLC, high-performance thin layer chromatography; PC, phosphatidylcholine; PE, phosphatidylethanolamine; PPAR, peroxisome proliferator-activated receptor; PTZ, pentylenetetrazol; PUFA, polyunsaturated fatty acids
} 
Table 1. Composition of fatty acids ( $\mathrm{g} / 100 \mathrm{~g})$ of LCP and palm oil that were administered by gavaging during $30 \mathrm{~d}$

\begin{tabular}{llcc}
\hline & & $\begin{array}{c}\text { LCP blend } \\
(\mathrm{g} / 100 \mathrm{~g})\end{array}$ & $\begin{array}{c}\text { Palm oil } \\
(\mathrm{g} / 100 \mathrm{~g})\end{array}$ \\
\hline Saturated & 24.24 & 49.5 \\
$\quad$ Palmitic acid & $\mathrm{C} 18: 0$ & 15 & 44 \\
Mono-unsaturated & & 23.5 & 39.5 \\
Oleic acid & $\mathrm{C} 18: 1(\mathrm{n}-9)$ & 14.8 & 38 \\
Polyunsaturated & & 52.26 & 11 \\
Linoleic acid & $\mathrm{C} 18: 2(\mathrm{n}-6)$ & 11.2 & 10 \\
$\alpha$-Linolenic acid & $\mathrm{C} 18: 3(\mathrm{n}-3)$ & 1.7 & 0.5 \\
Arachidonic acid & $\mathrm{C} 20: 4(\mathrm{n}-6)$ & 7.4 & \\
Eicosapentaenoic acid & $\mathrm{C} 20: 5(\mathrm{n}-3)$ & 5.5 & \\
Docosahexaenoic acid & $\mathrm{C} 22: 6(\mathrm{n}-3)$ & 8 & \\
\hline
\end{tabular}

A

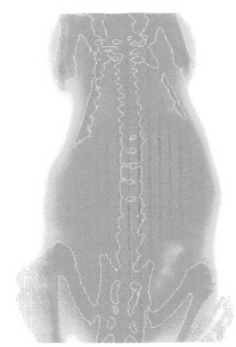

C

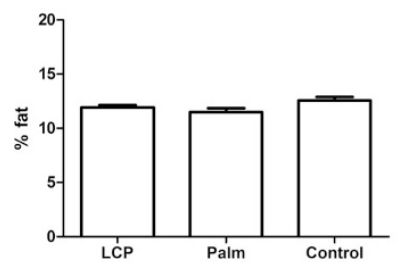

B

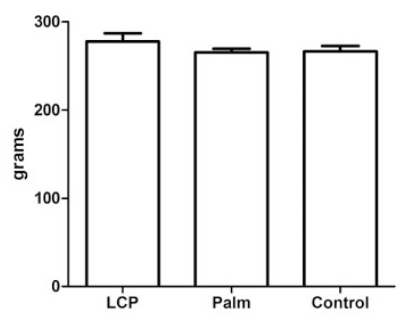

D

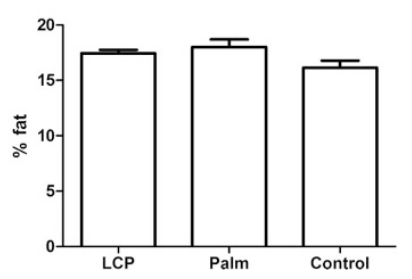

Figure 1. Nutritional status. (A) PIXImus densitometer scan of a studied rat. Body fat composition (\%) was comparable between groups at the start and the end of the study. Body weight measure at the end of the diet (D-30) $(B)$. Percentage of body fat $(C) 6 \mathrm{~d}$ before the diet onset (D-0) and $(D)$ the last day of the diet (D-30) in LCP $(n=11)$, palm $(n=11)$, and control $(n=10)$ groups measured with the PIXImus densitometer. Scan of an animal (upper left corner). Values are mean \pm SEM.

lished for ALA, LA, or LA/ALA mix (7). Rational design for new clinical trials should be based on preclinical data using oral administration and should include the type, the duration, and the amount of PUFA that afford anticonvulsant effects.

We recently showed that a 30-d-long oral administration of LA/ALA mix exhibited anticonvulsant properties in rats without impacting the composition of phospholipids in the neural cell membranes (11). In this study, we examined whether similar quantities of DHA/EPA possess anticonvulsant properties in rats.

\section{METHODS}

Animals. The experiments were performed on 4-wk-old male Wistar rats (100-120 g; Charles River, L'arbresle, France). The animals were housed six to eight per cage with an alternating 12-h light/dark cycle with lights on at $0700 \mathrm{~h}$. All procedures were approved by the Animal Research Committee of Lille University and were performed in agreement with the local, institutional, national, and international laws and regulations.

Treatments. All animals had been maintained on standard rodent chow and water ad libitum before they were put on the diet. Rats were weighted and divided into three diet treatment groups: (1) a standard diet containing $2.46 \%$ of PUFA (Harlan Teklad Global 2016, Gannat, France) (control group, $n=$ 10) (2); standard diet with daily oral supplementation (via gavage) of palm oil (palm group, $n=11$ ) (3); and standard diet with daily oral supplementation (via gavage) of a PUFA blend which provided $400 \mathrm{mg} / \mathrm{kg} / \mathrm{d}$ of DHA and 275 $\mathrm{mg} / \mathrm{kg} / \mathrm{d}$ of EPA [long chain polyunsaturated fatty acids capsules (LCP); SHS,
Liverpool, UK] (DHA/EPA group, $n=11$ ) (Table 1). The rats were maintained on the diet for $30 \mathrm{~d}$. Standard diet was served ad libitum. The LCP blend or the palm oil was administered by gavaging (p.o.) at $5 \mathrm{~g} / \mathrm{kg}$ of body weight/d. All rats were weighted at least twice a week.

Using these methods, we provided an additional amount of saturated fatty acids in the palm group and an additional amount of polyunsaturated fatty acids in the LCP group. The details are provided in Table 1. Dietary regimen of DHA/EPA was congruent with previously established anticonvulsant effects of ALA/LA (11). The palm oil was used as a control.

Pentylenetetrazol seizure threshold. A pentylenetetrazol (PTZ; Sigma Chemical Co-Aldrich, France) seizure threshold was examined between $0900 \mathrm{~h}$ and $0000 \mathrm{~h}, 30 \mathrm{~d}$ after the beginning of each dietary treatment. PTZ was dissolved in physiologic saline at $10 \mathrm{mg} / \mathrm{mL}$ and was infused with a syringe pump into the tail vein of freely moving rats at a constant rate of 2 $\mathrm{mL} / \mathrm{h}$. Time to the first forelimb myoclonus (i.e. bilateral forelimb myoclonus inducing a move of the forelegs from the ground) was used as the end point. Seizure threshold was expressed as total of $\mathrm{mg} / \mathrm{kg}$ of PTZ sufficient to induce forelimb myoclonus (control, $n=10$; palm, $n=11$; LCP, $n=11$ ) (23-25). The described seizure test has been commonly used in in vivo studies of anticonvulsant properties of PUFA (7), including our earlier experiments (11), and thus represents a good comparison assay.

Body composition. Six days before the diet onset (D-0) and on the last day (D-30) of the study, after PTZ threshold test, rats were anesthetized with chloral hydrate $(300 \mathrm{mg} / \mathrm{kg}$, i.p.) and scanned using a Lunar PIXImus densitometer (GE Lunar, Madison, WI; Fig. 1) for body composition measurement.

Lipid analysis. After the assessment of PTZ seizure threshold and body composition measurement, rats were killed by the overdose of pentobarbital (180 mg/kg i.p.). Blood samples were drawn by cardiac punctures, followed by the removal of the brains. Blood samples were centrifuged for $20 \mathrm{~min}$ at $3500 \mathrm{rpm}$ and $4^{\circ} \mathrm{C}$; plasma was separated and stored at $-80^{\circ} \mathrm{C}$. Brains (without cerebellum) were frozen in liquid nitrogen and stored at $-80^{\circ} \mathrm{C}$ [LCP $(n=11)$, palm $(n=11)$, and control $(n=10)]$.

Lipid extraction from the plasma and the homogenized brains was performed with chloroform/methanol as described earlier (26). About 300-600 $\mathrm{mg}$ of brain tissue and $200 \mu \mathrm{L}$ of serum were used for each analysis. Organic phase obtained after extraction was concentrated, and individual lipid classes within extract were separated by the one-dimension [plasma or twodimension (brain)] high-performance thin layer chromatography (HPTLC) (27). Briefly, HPTLC plate was developed with chloroform/methanol/16.5 N aqueous ammonia $65: 25: 5 \mathrm{vol} / \mathrm{vol} / \mathrm{vol}$, in the first dimension, followed by chloroform/acetone/methanol/acetic acid/water 3:4:1:1:0.5 vol/vol/vol/vol/ $\mathrm{vol}$ in the second dimension. Isolated phospholipid subclasses phosphatidylcholine (PC) and phosphatidylethanolamine (PE) were scraped off the HPTLC plate and trans-esterified in 14\% boron trifluoride-methanol (Alltech, code 18017, Alltech Biotechnologies, Carquefou, France) in a sealed vial in nitrogen atmosphere at $100^{\circ} \mathrm{C}$ for $45 \mathrm{~min}$ (28). The resulting fatty acid methyl-esters (FAME) were extracted with pentane, dehydrated over sodium sulfate, decahydrated $\left(\mathrm{Na}_{2} \mathrm{SO}_{4}, 10 \mathrm{H}_{2} \mathrm{O}\right)$, evaporated in a vacuum concentrator and prepared for gas chromatography (GC) by sealing pentane extract in nitrogen. FAME were separated and quantified by capillary GC using a gas chromatograph (HewlettPackard model 5890, Wilmington, DE) equipped with a $60 \mathrm{~m} / 0.32 \mathrm{~mm}$ DB-23 capillary column (J\&W Scientific, Folsom, CA) and flame-ionization detector. A time-programmed temperature from $170^{\circ} \mathrm{C}$ to $230^{\circ} \mathrm{C}$ was used. Individual fatty acids were identified by comparison of retention time with the known standard and quantified with an HP3396 integrator.

Statistical analysis. Data are expressed as mean \pm SEM. Statistical analysis was performed using Kruskal-Wallis test and Dunn post hoc analysis using GraphPad Prism 5.02 (San Diego, CA). Statistical significance was considered to be $p<0.05$.

\section{RESULTS}

Weight and body composition were comparable across groups and tends to increase from D-0 to D-30. During the dietary treatments, all animals remained visibly in good health and appeared well groomed. No differences were observed in the mean body fat component among LCP, palm, and control groups $6 \mathrm{~d}$ before the diet onset (D-0) and on day-30 (D-30, the last day of treatment; Fig. 1). Mean weights were also similar among the studied groups.

No differences were observed in PTZ seizure threshold among all groups. PTZ seizure thresholds (PTZth) were similar in all groups. PTZth values were as follows: LCP 
group, $43 \pm 1.5 \mathrm{mg} / \mathrm{kg}$; palm group, $44.4 \pm 1.4 \mathrm{mg} / \mathrm{kg}$; and control, $43.2 \pm 1.9 \mathrm{mg} / \mathrm{kg}$ (Fig. 2).

Plasma fatty acid composition was modified by the dietary treatments. We observed a modification of the fatty acid composition of the plasma phospholipids. Particularly, the increase in the n-3 PUFA component was observed in the LCP group when compared with other groups. This was evident with the increase of the n-3 PUFA and the decrease of the n-6/n-3 ratio (Fig. 3). Furthermore, we observed the increase of both DHA and EPA in the LCP group when compared with other groups (Fig. 3). No differences in arachidonic acid (AA), ALA, and LA were found among all the groups.

The fatty acid composition of the brain cell membranes was modified by the diets. We found the decrease in the composition in n-6 PUFA of the PE in the LCP group when compared with the control group, while no difference was observed between the palm versus the two other groups (Fig. 4). This decrease was not related to the component in AA of the PE group. We found the

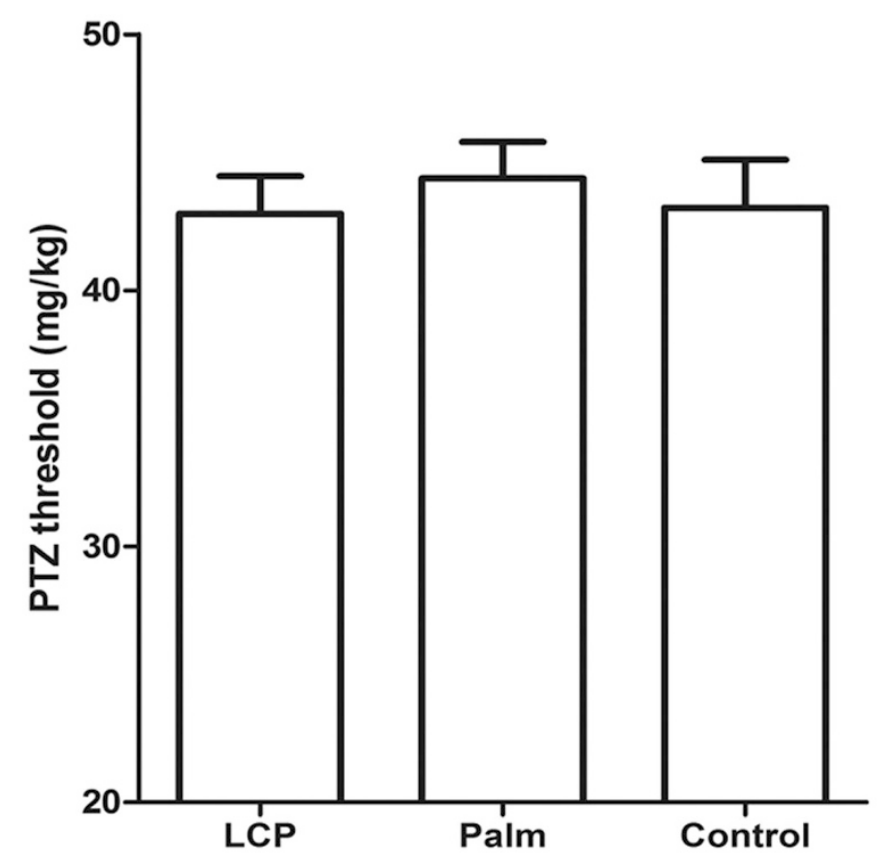

Figure 2. Effects of 30-d preventive treatments on myoclonic PTZ thresholds $(\mathrm{mg} / \mathrm{kg})$ in LCP $(n=11)$, palm $(n=11)$, and control $(n=10)$ groups. Values are mean \pm SEM. PTZ thresholds were similar among the groups. increase in the composition in AA of the PE in both LCP and palm groups compared with the control group (Fig. 4).

We did not observe an increase of n-3 PUFA, n-6 PUFA, or saturated fatty acid in the composition of the PC in the brain among the groups. However, control group was characterized by a higher level of EPA in the composition of PC (Fig. 5).

\section{DISCUSSION}

Daily oral supplementation with the mix of DHA/EPA did not exhibit an anticonvulsant effect in the PTZ threshold (LCP group). We excluded that the nutritional status could be a confounding factor in our study. Thirty days after the onset of the dietary treatment, the fatty acid composition of the plasma phospholipids was modified, which reflected the diet intake. We also observed modifications in the composition of the brain phospholipids. We noted the discrepancy between the present findings and the results of our previous study, which used the same amount of PUFA and the same experimental design. The difference between these two studies consisted in the type of PUFA in the gavage (LA/ALA in the previous and DHA/EPA in this study) (11). Our findings prompt modifications in the design of future preclinical trials of PUFA and particularly the examination of their anticonvulsant properties.

There is growing experimental evidence suggesting that PUFA may possess anticonvulsant properties (7). However, clinical trials, which provided various quantities of blends containing DHA and EPA, report conflicting results. In an open study, 21 patients with intractable epilepsy received $5 \mathrm{~g}$ n-3 PUFAs daily (46\% DHA, 18\% EPA, 1\% ALA) for 6 mo. Five patients completed the study with a reduction in both frequency and severity of seizures (22). In another, randomized study, the administration of an EPA/DHA mixture (10:7) led to the decrease of seizure frequency during the first 6-wk treatment period; however, the effect was not sustained (21). Bromfield et al. (20) failed to obtain seizure protection with an EPA/DHA (3:2) mixture, which was given for $12 \mathrm{wk}$. Several hypotheses may explain the discrepancies between the outcomes of animal studies and clinical trials (7). Therefore, before proceeding with the design of a new clinical trial, additional animal-based experiments are required.

Among the PUFA, only DHA is found in appreciable amounts in the brain and experimental data suggest its anticonvulsant

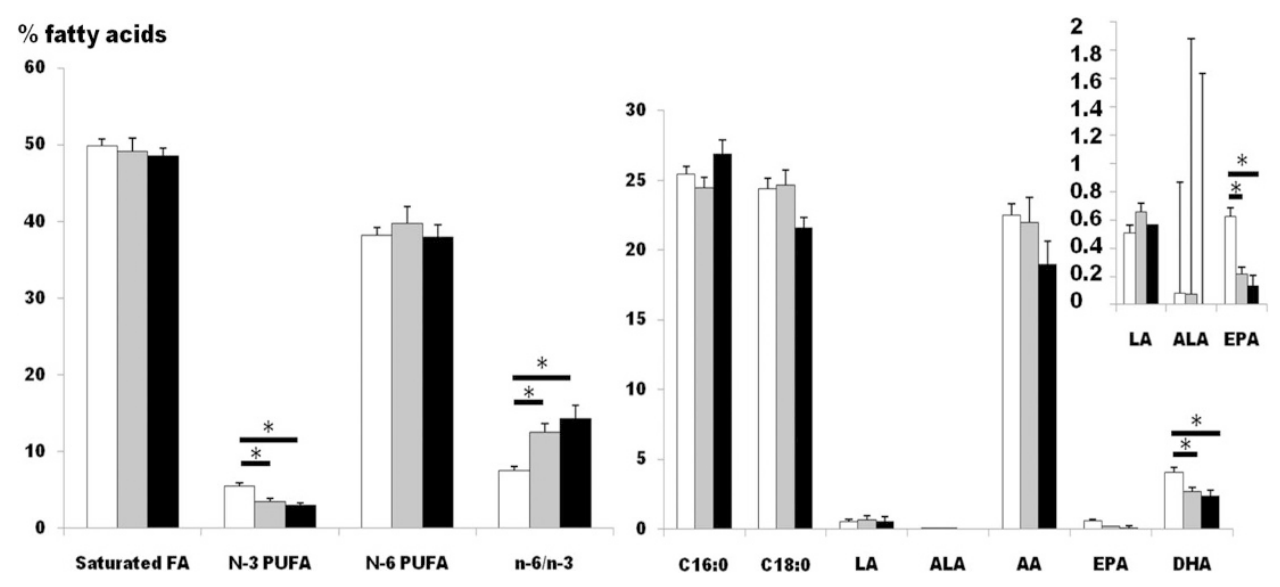

Figure 3. Plasma phosphatidylcholine fatty acid (FA) composition LCP $(\square)(n=$ $11)$, palm ( $\square)(n=11)$, and control $(\square)$ $(n=10)$. Results are expressed as \% of total lipids. We found an increase in the n-3 PUFA component in the LCP group that was related to an increase of DHA and EPA. $* p \leq 0.05$. 

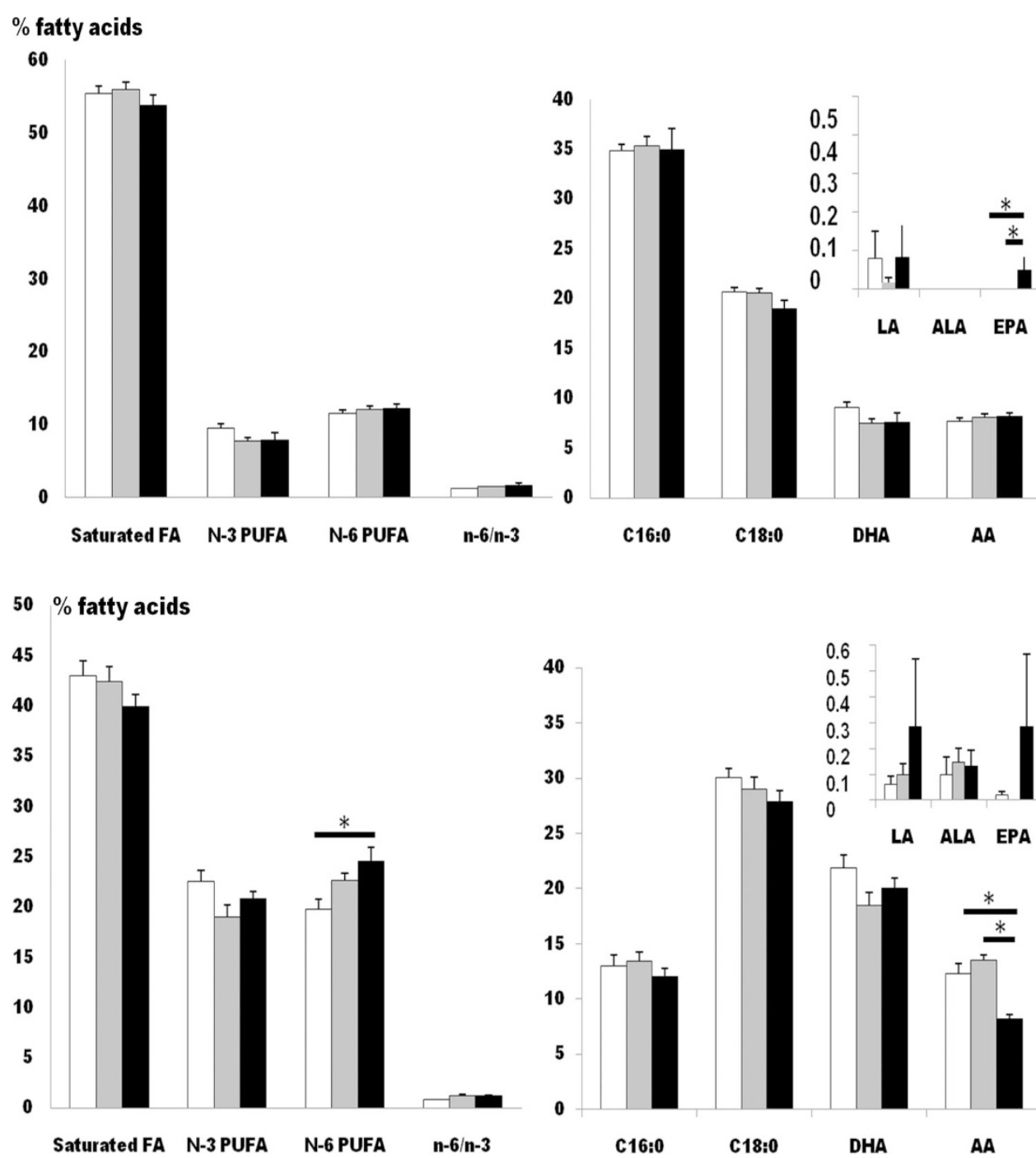

Figure 4. Fatty acids of phosphatidylethanolamine $(\mathrm{PE})$ fraction in the brain of $\operatorname{LCP}(\square)(n=11)$, palm $(\square)(n=11)$, and control (ם) $(n=10)$ groups. Results are expressed as $\%$ of total fatty acids. We also found the decrease of $n-6$ PUFA in the composition of PE in the LCP group unrelated to the modification of AA level. We found the increase in the composition in AA of the PE in both LCP and palm groups compared with the control group. $* p \leq 0.05$.
Figure 5. Fatty acids of phosphatidylcholine (PC) fraction in brain in the LCP $(\square)(n=11)$, palm $(\square)(n=11)$, and control ( $\square)(n=10)$ groups. Results are expressed as $\%$ of total fatty acids. We found a higher level of EPA in the composition of PC in the control group compared with the others. ${ }^{*} p \leq 0.05$. efficacy. It has been shown that fat- 1 mice (i.e. DHA mice that are genetically altered to produce elevated levels of DHA in the brain when they consume a normal diet) exhibit longer latency of PTZ-induced seizures when compared with the wild-type mice (29). More recently, it has been established that subcutaneous injection of $400 \mathrm{mg} / \mathrm{kg}$ of DHA is able to increase the latency of PTZ seizures (14). This effect was observed only when DHA was administered $1 \mathrm{~h}$ before PTZ injection. The anticonvulsant effect of this fatty acid was not observed when mice received a diet containing $6 \mathrm{~g}$ of DHA/ $\mathrm{kg}$ of diet (30). Using the amount of PUFA similar to that used by Taha et al. during $30 \mathrm{~d}$, we did not observe any anticonvulsant effect. We suggest that a longer duration of oral administration may result in the outcome similar to the one reported by Taha et al. (14). This may be due to the fact that systemic administration can rapidly modify the amount of FFA in the plasma, while upon oral administration longer time may be needed for FFA levels to reach a therapeutic level in the plasma (7).

In our study, the diet modification was responsible for a significant increase of n-3 PUFA in the plasma in the LCP group. This was related to the increase of both DHA and EPA (Fig. 3). It is interesting to note that the anticonvulsant effect of DHA was found only when it was provided by systemic injection. We did not observe any anticonvulsive effect of a mix of DHA/EPA upon their oral administration despite the increase of their plasma levels, as it was previously reported in mice (30). Therefore, the anticonvulsant efficacy of DHA may depend on the route of administration (i.e. injection versus oral). When administered orally, DHA is trapped in the chylomicron/ LDL pools. DHA probably does not appear in its unesterified (albumin-bound) form in plasma until the chylomicron/LDL pool is eventually saturated. Furthermore, DHA levels in the brain may increase slowly, even after unesterified DHA appears in the plasma. As an example, the half-life of DHA in the human brain is approximately $2.5 \mathrm{y}$ (31). The relationship between the different forms of PUFA in the plasma and their anticonvulsant effect should be further explored.

We found a higher level of EPA in the composition of PC in control animals compared with other groups. We observed the decrease of n-6 PUFA in the composition of PE in the LCP group although the increase in the composition in AA of the $\mathrm{PE}$ in both LCP and palm groups was comparable to that in the control group. However, these modifications were not related to any anticonvulsant effect. In previous studies, PUFA treatment exerted anticonvulsant effects in the absence of any modification of PUFA composition in the brain $(10,11)$. 
Despite the fact that DHA is considered to be a key factor in the anticonvulsant mechanism of action of PUFA, previous studies found no significant increase of DHA concentrations in brain membrane phospholipids $(10,11)$. The latter finding can be attributed to three possible causes. First, the lipid analysis was done postseizure, and seizures in turn could induce the loss of phospholipid DHA due to its release from the membrane. This was also the case in this study. Second, the gas-chromatographic assays used to measure esterified DHA levels in brain phospholipids could be not sensitive enough to detect small increases in the amount of brain DHA. It is also possible that the anticonvulsant properties of DHA are not linked to the modification of PUFA levels within the brain. Rather, seizure suppression could be a result of either binding of n-3 PUFAs to PPAR- $\alpha$ and/or PPAR $\gamma$ receptors or of anti-inflammatory effects of $n-3$ PUFAs (7). The suggestion that PPARs may be involved in seizure control is based on animal studies that have reported the increase of seizure thresholds by selective agonists of PPAR- $\alpha$ or PPAR- $\gamma$. For example, Fenofibrate (a potent inducer of PPAR $\alpha$ ) raised seizure threshold in the tail-infusion PTZ and pilocarpine seizure tests in rats (32). Maurois et al. (33) have also found that the PPAR- $\gamma$ ligand, FMOC-L-leucine, was protective against audiogenic seizures induced by magnesium deficiency in mice.

Using the same amount of DHA that was previously published, we failed to find any anticonvulsant effect. Insufficiently high dose can be excluded as a cause of DHA failure to modify PTZ threshold because daily oral DHA/EPA gavaging was accompanied by both plasma and CNS cell membrane modifications as described above. Furthermore, it should kept in mind that the administration of a LA/ALA mix over the same period and amount effectively increases PTZ seizure threshold (11).

In conclusion, further clinical trials should be preceded by preclinical studies that would clarify the amount and the duration of PUFA administration required to exhibit an anticonvulsant effect. Oral administration of PUFA is more relevant to clinical scenarios. It is also worth considering the use of PUFA with already established anticonvulsant effects, e.g. LA/ALA. Because of the dose dependence of anticonvulsant effect of PUFA (10), dose-curve studies should be factored into future clinical trials.

Acknowledgments. The authors thank Andrey Mazarati, M.D., Ph.D., for his helpful comments and SHS-Nutricia for a gift of LCP capsules.

\section{REFERENCES}

1. Picot MC, Baldy-Moulinier M, Daures JP, Dujols P, Crespel A 2008 The prevalence of epilepsy and pharmacoresistant epilepsy in adults: a population-based study in a Western European country. Epilepsia 49:1230-1238

2. Sander JW 2003 The epidemiology of epilepsy revisited. Curr Opin Neurol 16:165170

3. Coppola G, Veggiotti P, Cusmai R, Bertoli S, Cardinali S, Dionisi-Vici C, Elia M, Lispi ML, Sarnelli C, Tagliabue A, Toraldo C, Pascotto A 2002 The ketogenic diet in children, adolescents and young adults with refractory epilepsy: an Italian multicentric experience. Epilepsy Res 48:221-227

4. Neal EG, Chaffe H, Schwartz RH, Lawson MS, Edwards N, Fitzsimmons G, Whitney A, Cross JH 2008 The ketogenic diet for the treatment of childhood epilepsy: a randomised controlled trial. Lancet Neurol 7:500-506
5. Vining EP, Freeman JM, Ballaban-Gil K, Camfield CS, Camfield PR, Holmes GL, Shinnar S, Shuman R, Trevathan E, Wheless JW 1998 A multicenter study of the efficacy of the ketogenic diet. Arch Neurol 55:1433-1437

6. Freeman JM, Vining EP, Pillas DJ, Pyzik PL, Casey JC, Kelly LM 1998 The efficacy of the ketogenic diet-1998: a prospective evaluation of intervention in 150 children. Pediatrics 102:1358-1363

7. Taha AY, Burnham WM, Auvin S 2010 Polyunsaturated fatty acids and epilepsy. Epilepsia 51:1348-1358

8. Rabinovitz S, Mostofsky DI, Yehuda S 2004 Anticonvulsant efficiency, behavioral performance and cortisol levels: a comparison of carbamazepine (CBZ) and a fatty acid compound (SR-3). Psychoneuroendocrinology 29:113-124

9. Yehuda S, Carasso RL, Mostofsky DI 1994 Essential fatty acid preparation (SR-3) raises the seizure threshold in rats. Eur J Pharmacol 254:193-198

10. Taha AY, Filo E, Ma DW, McIntyre BW 2009 Dose-dependent anticonvulsant effects of linoleic and alpha-linolenic polyunsaturated fatty acids on pentylenetetrazol induced seizures in rats. Epilepsia 50:72-82

11. Porta N, Bourgois B, Galabert C, Lecointe C, Cappy P, Bordet R, Vallee L, Auvin S 2009 Anticonvulsant effects of linolenic acid are unrelated to brain phospholipid cell membrane compositions. Epilepsia 50:65-71

12. Blondeau N, Widmann C, Lazdunski M, Heurteaux C 2002 Polyunsaturated fatty acids induce ischemic and epileptic tolerance. Neuroscience 109:231-241

13. Voskuyl RA, Vreugdenhil M, Kang JX, Leaf A 1998 Anticonvulsant effect of polyunsaturated fatty acids in rats, using the cortical stimulation model. Eur J Pharmacol 341:145-152

14. Taha AY, Jeffrey MA, Taha NM, Bala S, Burnham WM 2010 Acute administration of docosahexaenoic acid increases resistance to pentylenetetrazol-induced seizures in rats. Epilepsy Behav 17:336-343

15. Vreugdenhil M, Bruehl C, Voskuyl RA, Kang JX, Leaf A, Wadman WJ 1996 Polyunsaturated fatty acids modulate sodium and calcium currents in CA1 neurons. Proc Natl Acad Sci USA 93:12559-12563

16. Xiao YF, Kang JX, Morgan JP, Leaf A 1995 Blocking effects of polyunsaturated fatty acids on $\mathrm{Na}+$ channels of neonatal rat ventricular myocytes. Proc Natl Acad Sci USA 92:11000-11004

17. Xiao YF, Gomez AM, Morgan JP, Lederer WJ, Leaf A 1997 Suppression of voltage-gated L-type $\mathrm{Ca} 2+$ currents by polyunsaturated fatty acids in adult and neonatal rat ventricular myocytes. Proc Natl Acad Sci USA 94:4182-4187

18. Taha AY, Ryan MA, Cunnane SC 2006 Markedly raised intake of saturated and monounsaturated fatty acids in rats on a high-fat ketogenic diet does not inhibit carbon recycling of 13C-alpha-linolenate. Lipids 41:933-935

19. Demar JC, Jr., Ma K, Chang L, Bell JM, Rapoport SI 2005 alpha-Linolenic acid does not contribute appreciably to docosahexaenoic acid within brain phospholipids of adult rats fed a diet enriched in docosahexaenoic acid. J Neurochem 94:1063-1076

20. Bromfield E, Dworetzky B, Hurwitz S, Eluri Z, Lane L, Replansky S, Mostofsky D 2008 A randomized trial of polyunsaturated fatty acids for refractory epilepsy. Epilepsy Behav 12:187-190

21. Yuen AW, Sander JW, Fluegel D, Patsalos PN, Bell GS, Johnson T, Koepp MJ 2005 Omega-3 fatty acid supplementation in patients with chronic epilepsy: a randomized trial. Epilepsy Behav 7:253-258

22. Schlanger S, Shinitzky M, Yam D 2002 Diet enriched with omega-3 fatty acids alleviates convulsion symptoms in epilepsy patients. Epilepsia 43:103-104

23. Auvin S, Shin D, Mazarati A, Sankar R 2006 The utility of testing pentylenetetrazol threshold. Epilepsia 47:662-663

24. Pollack GM, Shen DD 1985 A timed intravenous pentylenetetrazol infusion seizure model for quantitating the anticonvulsant effect of valproic acid in the rat. J Pharmacol Methods 13:135-146

25. Löscher W, Hönack D, Fassbender CP, Nolting B 1991 The role of technical, biological and pharmacological factors in the laboratory evaluation of anticonvulsant drugs. III. Pentylenetetrazole seizure models. Epilepsy Res 8:171-189

26. Bligh EG, Dyer WJ 1959 A rapid method of total lipid extraction and purification. Can J Biochem Physiol 37:911-917

27. Galabert C, Jacquot J, Zahm JM, Puchelle E 1987 Relationships between the lipid content and the rheological properties of airway secretions in cystic fibrosis. Clin Chim Acta 164:139-149

28. Morrison WR, Smith LM 1964 Preparation of fatty acid methyl esters and dimethylacetals from lipids with boron fluoride-methanol. J Lipid Res 5:600-608

29. Taha AY, Huot PS, Reza-Lopez S, Prayitno NR, Kang JX, Burnham WM, Ma DW 2008 Seizure resistance in fat- 1 transgenic mice endogenously synthesizing high levels of omega-3 polyunsaturated fatty acids. J Neurochem 105:380-388

30. Willis S, Samala R, Rosenberger TA, Borges K 2009 Eicosapentaenoic and docosahexaenoic acids are not anticonvulsant or neuroprotective in acute mouse seizure models. Epilepsia 50:138-142

31. Umhau JC, Zhou W, Carson RE, Rapoport SI, Polozova A, Demar J, Hussein N, Bhattacharjee AK, Ma K, Esposito G, Majchrzak S, Herscovitch P, Eckelman WC, Kurdziel KA, Salem N Jr 2009 Imaging incorporation of circulating docosahexaenoic acid into the human brain using positron emission tomography. J Lipid Res 50:1259-1268

32. Porta N, Vallee L, Lecointe C, Bouchaert E, Staels B, Bordet R, Auvin S 2009 Fenofibrate, a peroxisome proliferator-activated receptor-alpha agonist, exerts anticonvulsive properties. Epilepsia 50:943-948

33. Maurois P, Rocchi S, Pages N, Bac P, Stables JP, Gressens P, Vamecq J 2008 The PPARgamma agonist FMOC-l-leucine protects both mature and immature brain. Biomed Pharmacother 62:259-263 